Meta

Journal des traducteurs

Translators' Journal

\title{
L'enseignement de la traduction en Algérie
}

\section{Aïcha Aïssani}

Volume 45, numéro 3, septembre 2000

La traduction dans le monde arabe

URI : https://id.erudit.org/iderudit/001864ar

DOI : https://doi.org/10.7202/001864ar

Aller au sommaire du numéro

Éditeur(s)

Les Presses de l'Université de Montréal

ISSN

0026-0452 (imprimé)

1492-1421 (numérique)

Découvrir la revue

Citer cet article

Aïssani, A. (2000). L'enseignement de la traduction en Algérie. Meta, 45(3), 480-490. https://doi.org/10.7202/001864ar

\section{Résumé de l'article}

La situation linguistique en Algérie, qui se caractérise par un bilinguisme arabe-français bien enraciné dans l'histoire, a été marquée par des pratiques d'enseignement différentes selon les périodes historiques et par une politique d'arabisation à partir de 1970. C'est dans ce cadre que s'inscrit la formation des traducteurs en Algérie telle qu'elle se dégage des cursus, de la méthodologie adoptée et des représentations que se font enseignants et apprenants de leur statut respectif. 


\title{
L'enseignement de la traduction en Algérie
}

\author{
AÏCHA AÏSSANI \\ Université d'Alger, Alger, Algérie
}

\begin{abstract}
RÉSUMÉ
La situation linguistique en Algérie, qui se caractérise par un bilinguisme arabe-français bien enraciné dans l'histoire, a été marquée par des pratiques d'enseignement différentes selon les périodes historiques et par une politique d'arabisation à partir de 1970. C'est dans ce cadre que s'inscrit la formation des traducteurs en Algérie telle qu'elle se dégage des cursus, de la méthodologie adoptée et des représentations que se font enseignants et apprenants de leur statut respectif.
\end{abstract}

\section{ABSTRACT}

The linguistic situation in Algeria is characterized by an Arabic-French bilinguism wellrooted in the history of the country, where teaching practices varied according to historical periods and to a policy of arabization that started in 1970. It is within this framework that translation training is practised as can be seen by the programmes and the methodology chosen and by the way teachers and learners view their respective status.

\section{MOTS-CLÉS/KEYWORDS}

enseignement, traduction, Algérie, formation, traducteur

\section{Introduction}

Le passé historique récent de l'Algérie fait que ce pays s'est trouvé et se trouve encore aujourd'hui confronté à un bilinguisme (français/arabe) qui s'impose de fait. Des institutions relevant de la traduction devaient dès lors être indispensables: la formation du traducteur et a fortiori l'enseignement de la traduction s'érigeaient en contraintes incontournables.

Un survol de l'enseignement de la traduction en Algérie, depuis l'occupation française à nos jours, nous permettra d'apprécier quelque peu la situation de ce mode de communication

\section{Avant 1962: Les lycées franco-musulmans}

Les écoles coraniques et la medersa qui constituaient à l'époque deux institutions informelles pour l'administration française et qui se réservaient respectivement l'apprentissage du Coran, et l'enseignement de la langue arabe par des autochtones monolingues formés dans les zaouias furent supplantées par les lycées franco-musulmans nés le 30 septembre 1850 sous l'autorité de l'administration française, ils sont une reconversion de l'École Supérieure Musulmane née en 1836 à Constantine.

Ces lycées se spécialisaient dans la formation de bilingues français-arabe et inversement. Ainsi le premier lycée franco-musulman fut installé d'abord à Médéa puis à Blida et enfin en 1859 à Alger, suivirent Tlemcen et Constantine. A la fin de leur cursus, les diplômés de ces lycées franco-musulmans étaient censés enseigner la langue 
arabe dans les lycées: l'enseignement de la langue arabe se limitant à deux principales matières: la version et le thème:

La version étant la traduction de la langue étrangère vers la langue de base. Or à cette époque la version autrement dit la traduction de la langue étrangère vers la langue maternelle se faisait pour comble d'ironie pour les Algériens de la langue arabe vers la langue française. La langue française était considérée alors comme langue maternelle des Algériens.

Le thème, lui, est la traduction de la langue de base vers la langue étrangère autrement dit de la langue française vers la langue arabe.

$\mathrm{Vu}$ sous l'angle de l'apprentissage des langues, on est en droit de penser que la version et le thème sont essentiellement une opération de transcodage, ce qui nous éloigne de la véritable traduction. Mais est-il pertinent au niveau du scolaire de prétendre à la véritable traduction?

En effet sans vouloir prétendre à la véritable traduction, la traduction scolaire a a priori ses avantages, ce qui nous pousse à adhérer au point de vue de L. El Foul (1996: 43):

Ce qui est visé à travers la version et le thème, c'est la connaissance, par la comparaison des structures grammaticales des langues dont il s'agit. La traduction permet de prendre conscience des différences de structure entre la langue maternelle et les langues étrangères. Mieux que la présentation directe de ces structures à l'intérieur de chaque langue (exposé des règles et exercices d'application, exercices d'élocution, de rédaction, etc.), la traduction (comparaison, prise de conscience des différences) permet de caractériser chaque langue, c'est-à-dire approfondir la saisie des difficultés, et donc d'accroître la capacité de les résoudre.

\section{2: La langue arabe comme langue nationale}

En 1962, l'Algérie indépendante fut proclamée. La langue française occupait alors toujours une place prépondérante dans tous les secteurs et notamment dans le système éducatif où elle était obligatoirement langue d'enseignement. Aussi parmi les problèmes de l'heure (dès 1962), la politique linguistique fut l'une des préoccupations majeures des instances gouvernementales. C'est alors que restaurer la langue arabe dans ses droits, autrement dit comme langue nationale, ne pouvait que conforter un consensus national.

La langue arabe devenue officiellement langue nationale dans un paysage fortement marqué par la langue française (structures étatiques et formation des cadres susceptibles de prendre la relève à l'indépendance) sa mise en place relevait du défi. L'urgence était donc de trouver une institution capable de pallier, même à long terme, ce déficit.

L'initiative est venue de l'UNESCO: créer une école supérieure d'interprètes et de traducteurs à l'image de l'École Supérieure d'Interprètes et de Traducteurs de l'Université de Paris. C'est ainsi qu'en 1963 à Alger est fondée l'école Supérieure de Traduction et d'Interprétariat.

Trois combinaisons étaient assurées:

arabe - français - anglais

arabe - français - espagnol

arabe - français - allemand 
Une spécialité appelée «interprétation simultanée» était assurée pour former des interprètes de conférence.

\section{Dès 1970 : la politique d'arabisation}

La politique d'arabisation s'était intensifiée dès 1970 mettant alors en évidence un besoin pressant de personnel qualifié pour la traduction des textes officiels.

Le potentiel formé dans les lycées franco-musulmans allait être sollicité pour entreprendre dans les meilleures conditions l'arabisation de l'administration.

Pour ce faire, le Ministère de l'Intérieur crée des bureaux de traduction au niveau de chaque département ministériel. Ces bureaux étaient chargés de traduire les textes officiels de la langue française vers la langue arabe à des fins de publication dans le journal officiel. Dès lors tout texte officiel devait être obligatoirement en version arabe.

Par ailleurs d'autres besoins se sont faits sentir au niveau du Ministère des Affaires Étrangères, à certains niveaux des renseignements généraux et au Ministère de l'Intérieur.

\section{Sur le plan international}

Des concours se sont ouverts au niveau de l'OUA (Organisation de l'Unité Africaine), de la Ligue Arabe, des Nations Unies et du Bureau de Genève. Nous comptons actuellement au niveau de ces institutions plusieurs interprètes algériens.

\section{L'Université et la traduction}

Des cours de post-graduation ont été prévus pour l'encadrement des travaux de recherche. Par ailleurs des cours sont dispensés aux futurs licenciés. Les débouchés offerts à ces diplômés se restreignent généralement à la fonction de journaliste à l'APS (Agence Presse Service), à des quotidiens nationaux et aux différents départements de l'information et de la culture.

Aujourd'hui, un doctorat d'État est prévu dans le cadre de l'Institut d'Interprétariat et de Traduction en perspective de la création des facultés des Sciences Humaines qui verra le jour ce premier semestre 1999 à Alger et dont l'une des facultés engloberait l'Institut de la Littérature Arabe, l'Institut des Langues Étrangères et l'Institut d'Interprétariat et de Traduction.

Les titulaires du doctorat d'État pourront alors encadrer les travaux de recherche des magistères et des doctorats d'État.

Le nombre des étudiants en traduction allant croissant, les pouvoirs publics se sont vus dans l'obligation de créer deux départements de traduction à Oran et à Annaba. Des velléités pour créer un troisième à Batna. Une particularité pourtant caractérisera les deux départements de traduction d'Oran et de Annaba.

À Oran, on mettra davantage l'accent sur la combinaison arabe-français-espagnol étant donné que la communauté espagnole est très importante dans cette région. Par contre, on mettra l'accent sur la combinaison arabe-français-italien à Annaba où une communauté italienne prédomine. 


\section{Au niveau de la concertation des pays arabes}

Plusieurs universités arabes ont demandé la collaboration de l'Université d'Alger pour créer une école d'interprètes-traducteurs à Amen en Jordanie et une école similaire à Damas en Syrie.

La Ligue Arabe aurait choisi Alger mais, jusqu'à l'heure actuelle, cette initiative est restée à l'état de projet.

Après ce survol de la traduction, il est permis de penser que l'Algérie possède des traditions en matière de traduction. Pour s'en convaincre, il suffit de défiler le cours de l'histoire de l'Algérie; en effet, depuis l'Antiquité à nos jours, l'Algérien fut de tout temps confronté à la traduction d'une langue étrangère: héritage des multiples invasions; pour l'exemple, lorsque les premiers éléments de recensement de la population algérienne concourraient à la dénomination de certains chefs de famille par la profession, il ne faut pas s'étonner de voir le Tordjman apparaître en tête de liste des métiers hérités de l'empire Ottoman. En matière de justice, les Algériens ont connu le traducteur au sens de greffier et l'interprète au sens d'avocat.

Toujours en matière de justice, un aspect particulier de la traduction: «l'emprunt». Ainsi les premiers termes existants jusqu'à présent tels que la rahnia, la Frédha, le habous, etc. pénètrent le langage juridique par la Tardjama.

Ce besoin chronique de traduction a-t-il suscité en dehors de pratiques ponctuelles, la création de véritables institutions devant prendre en charge ce mode de communication?

Pour répondre à cette question, tournons-nous vers les structures :

L'École Supérieure de Traduction et d'Interprétariat fondé à Alger en 1963 devaitelle être à l'époque la panacée?

- Qu'en est-il également de la section de traduction et d'interprétariat fondée en 1971 et rattachée au département des Langues Étrangères de la faculté des Lettres et Sciences Humaines; puis du Département de Traduction et d'Interprétariat fondé en 1975 et rattaché l'Institut des Langues Étrangères?

- Qu'en est-il également du sort de l'Académie Houari Boumediene qui vraisemblablement était amenée à s'occuper de traduction à travers de l'étude de la terminologie?

Enfin le centre de traduction et de terminologie arabe fondé à Alger (décret du 17 avril 1980 et paru au journal officiel du 27 mai 1986) et qui devait s'atteler à la lourde tâche de recensement, d'harmonisation et de traduction, tout en promouvant la reconversion de certains enseignants vers la langue arabe a-t-il été opérationnel?

Ce survol de la situation linguistique et des multiples tentatives des instances gouvernementales pour créer des institutions susceptibles de contribuer à l'aboutissement de la décision politique d'arabisation montrent que le problème de la traduction est patent.

Qu'en est-il aujourd'hui où le processus d'arabisation (Ordonnance n ${ }^{\circ}$ 96-30 du 21/12/1996 modifiant et complétant la loi $\mathrm{n}^{\circ}$ 91-05 du 30 Joumada Ethania 1411 correspondant au 16 janvier 1991 portant généralisation de l'utilisation de la langue arabe) étendu à tous les secteurs de la vie politique socio-économique et culturelle a atteint son point de non-retour?

Notre propos dans cet article est donc d'essayer d'apporter un éclairage sur l'enseignement de la traduction en Algérie. 
Pour cela, nous avons porté notre choix sur une institution, la plus ancienne et $a$ priori la plus efficiente: l'Institut d'Interprétariat et de Traduction de l'Université d'Alger.

\section{Présentation}

L'Institut d'Interprétariat et de Traduction s'est vu créé grâce au décret n $84-209$ du 13/08/1985 portant sur l'organisation et la gestion de l'Université d'Alger.

C'est le prolongement de:

- l'École Supérieure de Traduction et d'Interprétariat fondée en 1963;

- la section de traduction et d'interprétariat fondée en 1971 et rattachée au Département des Langues Étrangères de la Faculté des Lettres et Sciences Humaines;

- du Département de Traduction et Interprétariat fondé en 1975 et rattaché à l'Institut des Langues Étrangères.

L'Institut prépare les études aux spécialités et langues suivantes:

\section{Spécialités}

L'Institut forme:

\subsection{Au niveau de la licence:}

- des traducteurs professionnels;

- des interprètes professionnels.

\subsection{Au niveau du magister:}

- des assistants universitaires en traduction et dans les matières qui s'y rattachent.

\section{Les langues au programme}

3.1. La langue de base: l'arabe.

3.2. Les langues de travail: le français, l'allemand, l'espagnol et l'anglais.

3.3. La langue complémentaire: l'italien.

\section{Préparation à la licence}

\subsection{Durée des études}

Les études pour l'obtention d'une licence en traduction et interprétariat durent quatre ans.

\subsection{Conditions d'accès à l'Institut :}

- le candidat doit être titulaire du baccalauréat (lettres);

- il doit réussir au concours d'entrée.

\subsection{Le concours d'accès}

Le concours d'entrée à l'Institut est fixé au mois de septembre de chaque année et ouvert aux bacheliers de séries lettres, langues étrangères et sciences. Pour chaque candidat, on procède à un examen approfondi du relevé de notes du baccalauréat en littérature et langues étrangères principalement.

\subsection{Inscription définitive}

Si le candidat est admis à l'Institut, il doit constituer un dossier d'inscription comprenant des fiches de renseignements à retirer au secrétariat de l'Institut et sur lesquelles il doit mentionner les indications demandées en y joignant les pièces requises.

\subsection{Transfert}

Les étudiants inscrits en $2^{\mathrm{e}}$ année de Licence, toutes filières confondues, peuvent prétendre à un transfert à l'Institut d'Interprétariat et de Traduction dans les mêmes conditions d'accès que les bacheliers. Ils sont admis en première année sur avis d'un conseil pédagogique après test en langue étrangère. 


\section{Programme d'études au niveau de la licence}

5.1. Première année

101: Sociologie

102: Linguistique

111: Langue A: pratique de la langue écrite: Exercices de compréhension et d'expression.

112: Langue A: pratique de la langue orale: Exercices de compréhension et d'expression.

113: Langue A: grammaire et exercices d'application.

$1^{\text {re }}$ : Langue étrangère:

121: Langue B: pratique de la langue écrite: Exercices de compréhension et d'expression.

122: Langue B: pratique de la langue orale: Exercices de compréhension et d'expression.

123: Langue B : grammaire: théorie et exercices d'application. Exercices de compréhension et d'expression.

$2^{\mathrm{e}}$ : Langue étrangère:

131: Langue B: pratique de la langue écrite: Exercices de compréhension et d'expression.

132: Langue B: pratique de la langue orale: Exercices de compréhension et d'expression.

133: Langue B: grammaire: théorie et exercices d'application.

\subsection{Deuxième année}

201: Initiation à l'économie politique

202: Initiation au droit

211: Langue A: pratique de la langue écrite: Exercices de compréhension et d'expression.

212: Langue A: pratique de la langue orale: Exercices de compréhension et d'expression.

213: Langue A: grammaire: théorie et exercices d'application.

221: Langue B: pratique de la langue écrite: Exercices de compréhension et d'expression.

223: Langue B: grammaire: théorie et exercices d'application.

231: Langue B: pratique de la langue écrite: Exercices de compréhension et d'expression $(+202 / 206)$

232: Langue B: pratique de la langue orale: Exercices de compréhension et d'expression (205)

233: Langue B: grammaire: théorie et exercices d'application.

241: Exercices de traduction entre les langues A et B.

242: Exercices de traduction entre les langues A et B.

245: (à option) Traduction B. .B.

Traductologie.

\subsection{Troisième année}

301: Encyclopédie de la traduction

311: Langue A: pratique de la langue écrite et orale.

312: Langue A: structure politique, économique, sociale et culturelle de l'Algérie et du monde arabe.

321: Langue B: pratique de la langue écrite et orale.

323: Stylistique comparée des langues A et B.

331: Langue B' : pratique de la langue écrite et orale.

332 : Langue B' : structure politique, économique, sociale et culturelle du ou des pays de la langue d'études 
333: Stylistique comparée des langues A et B'.

341: Exercices de traduction de la langue A vers la langue B (textes généraux).

342: Exercices de traduction de la langue $B$ vers la langue A (textes généraux).

343: Exercices de traduction de la langue A vers la langue B' (textes généraux).

344: Exercices de traduction de la langue B' vers la langue A (textes généraux).

351: Techniques de l'interprétation entre les langues $A$ et $B$ (textes généraux).

352: Techniques de l'interprétation entre les langues A et B' (pour les interprètes).

345: (à option) Exercices de traduction entre les langues B et B' (textes généraux).

\subsection{Quatrième année}

411: Langue A: pratique de la langue écrite et orale.

421: Langue B: pratique de la langue écrite et orale.

422: Langue B: structures politiques et économiques de la langue d'étude.

431: Langue B': pratique de la langue écrite et orale.

461 : Stage pratique.

462: Mémoire de fin d'études.

Pour les traducteurs :

441 : Traduction de la langue A vers la langue B (textes en langue de spécialité) (317/ S7).

442: Traduction de la langue B vers la langue A (textes en langue de spécialité).

443: Traduction de la langue A vers la langue B' (textes en langue de spécialité).

444: Traduction de la langue B' vers la langue A (textes en langue de spécialité).

451: Techniques de l'interprétation entre les langues A et B.

452: Techniques de l'interprétation entre les langues A et B'.

445: (à option) Traduction entre les langues B et B'.

Pour les interprètes:

453: Interprétation consécutive entre les langues A et B.

454: Interprétation consécutive entre les langues A et B'.

455: Interprétation simultanée de la langue A vers la langue B.

456: Interprétation simultanée de la langue B v ers la langue A.

457: Interprétation simultanée de la langue A vers la langue B'.

458: Interprétation simultanée de la langue B’ vers la langue A.

446: Traduction entre les langues A et B (textes en langue de spécialités).

447: Traduction entre les langues A et B' (textes en langue de spécialité).

459: (à option) Interprétation entre les langues B et B'.

\section{Préparation au Magister}

\subsection{Conditions d'accès :}

- être titulaire de la licence en traduction et interprétariat (ou d'un diplôme équivalent);

- avoir réussi au concours d'accès.

\subsection{Langues d'examen}

Le candidat doit subir un examen dans les deux langues programmées dans l'une des filières suivantes:

- arabe-français;

- arabe-anglais;

- arabe-espagnol.

\subsection{Durée des études}

Les études durent deux ans, durant lesquels les étudiants approfondissent leurs connaissances, s'exercent aux méthodes de recherche puis sont chargés de préparer un mémoire qui leur permettra d'enseigner à l'Université. 


\section{Programme d'étude au niveau du Magister}

\subsection{Magister de traduction}

7.1.1. Première année:

- Traduction de la langue étrangère vers la langue arabe et vice-versa;

- Stylistique comparée;

- Linguistique, lexicologie et lexicographie;

- Méthodologie;

- Théorie de la traduction;

- Renforcement du niveau en langue étrangère.

7.1.2. Deuxième année:

- Préparation du mémoire (en traduction et dans les disciplines qui s'y rattachent).

- Séminaires de recherche.

\subsection{Magister de traduction automatique}

7.2.1. Première année:

- Traduction de la langue arabe vers la langue étrangère et vice-versa;

- Stylistique comparée;

- Linguistique et syntaxe;

- Linguistique: lexicologie et lexicographie;

- Méthodologie;

- Théorie de la traduction;

- Linguistique formaliste;

- Informatique.

7.2.2. Deuxième année:

- Préparation du mémoire en traduction automatique et dans les disciplines qui s'y rattachent

- Séminaires de recherche.

\section{Le statut de la traduction}

Il n'existe pas à l'heure actuelle de statut de la traduction. En Algérie seul le statut de l'enseignant traducteur ou de l'enseignant interprète existe. Celui-ci est régi par les statuts de la fonction publique.

La carrière de ces deux types d'enseignants se calque sur le parcours de l'enseignant universitaire. Autrement dit, il sera assistant, maître-assistant, chargé de cours, maître de conférence et enfin professeur en fonction des diplômes qu'il aura obtenus.

Il n'y a pas de docteur en traduction (le travail sur la recherche en traduction est absent); l'assistant-traducteur se voit obligé de se reconvertir dans la discipline la plus proche de la traduction, en l'occurrence la linguistique pour entamer un travail de recherche. Généralement c'est la linguistique contrastive qui en est privilégiée. D'autres possibilités s'offrent à ce diplômé en traduction: il peut ouvrir un bureau de traduction si toutefois il est agrée par le ministère de la Justice, lequel fixe ses honoraires officiellement (voir le Journal officiel). Son activité se limitera à la traduction:

- d'actes judiciaires;

- des études notariales;

- de la traduction des diplômes étrangers;

- des expertises de tous genres. 


\section{Les langues utilisées}

Nous rappellerons pour mémoire les trois combinaisons déjà citées:

arabe - français - anglais

arabe - français - espagnol

arabe - français - allemand

\section{Les branches concernées}

L'École d'Interprétariat et de Traduction ne prévoit pas de spécialisation, le diplômé étant appelé à se former en fonction de son itinéraire professionnel.

En cours, la traduction tente de toucher à toutes les disciplines, c'est la traduction des thèmes aussi variés que possible qui donne la compétence nécessaire au traducteur.

\section{La méthodologie}

Aucune théorie d'ensemble de la traduction n'ayant été à ce jour "achevée», ce sont les études effectuées en matière de linguistique, de stylistique, de poétique, de communication et de traduction qui vont apporter les instruments d'analyse à la traduction.

Pour l'enseignant il n'existe pas de recettes en traduction: traduire, c'est poser préalablement et indéniablement le principe général du respect du message à différents niveaux:

- niveau quantitatif: respect de la somme d'informations contenue dans le message et que l'auteur veut effectivement transmettre;

- fidélité au style: respect du rythme et de la mélodie;

- fidélité au sens, ce qui présuppose:

a. d'un point de vue interne, des interrogations sur le cotexte et sur le contexte ainsi que de leurs implications d'ordre non seulement linguistique mais aussi cognitif.

b. d'un point de vue externe, une connaissance appropriée de l'idéologie politique, culturelle et même religieuse à laquelle adhère l'auteur.

Nous illustrerons cette partie méthodologie par un exemple concret: le texte à traduire tel qu'il est proposé à l'étudiant aux travaux dirigés de traduction générale.

De fait, le texte, en pédagogie de la traduction, est l'occasion d'un apprentissage actif. Il est de surcroît prétexte à l'acquisition d'une méthode de traitement (qui, à long terme et dans sa phase achevée, devra être complètement assimilée par le traducteur professionnel). Cette méthode de traitement est susceptible d'être généralisée à tous les types de textes (informatifs, argumentatifs, narratifs, descriptifs, etc.). Ceci implique que soient pris en considération le type de textes à traduire et, subséquemment, la progression envisagée dans le cursus. Le texte choisi (il peut l'être aussi bien par l'enseignant que par l'étudiant; certains enseignants préfèrent laisser le choix aux étudiants) est généralement «l'article de presse», document authentique traitant d'un problème d'actualité, texte beaucoup plus motivant que le texte didactique pris dans un manuel.

Un travail en commun sur le lexique, la syntaxe, l'articulation du texte et enfin le style est entamé; les divers choix qui se présentent devront alors être dûment expli- 
cités et argumentés pour aboutir à l'élaboration d'un texte supposé définitif mais constamment perfectible. En effet, un nouveau texte en langue cible ne peut prétendre à l'unicité, car il n'est l'expression que d'une seule reformulation supposée adéquate parmi le potentiel infini de textes susceptibles d'être issus du texte source.

Pour L. El Foul (1993: 120), «les deux capacités maîtresses du traducteur sont l'analyse et la comparaison ».

Cette considération implique que le souci majeur et impératif de l'enseignant est de développer chez l'étudiant des réflexes d'analyse du discours et de construction de sens, construction de sens qui est fonction d'un nombre important de variables aussi bien linguistiques qu'extra-linguistiques.

La tâche n'est pas aisée; traduire présuppose une grande dextérité en matière de performances multiples; le bon traducteur est en même temps linguiste (grammairien, phonéticien, sémanticien) et en possession d'une vaste culture.

Les représentations du traducteur et les difficultés évidentes qu'il éprouve, qu'il se trouve d'un côté ou de l'autre de la chaire (enseignant ou étudiant), en sont la preuve.

\section{Les représentations du corps enseignant}

De l'avis général, les enseignants se représentent la fonction de traducteur comme étant une fonction très noble puisqu'elle permet de rapprocher des personnes de langue et de culture différentes. Elle leur permet ainsi de se comprendre et de dissiper éventuellement leurs différents.

C'est également une fonction exaltante pour celui qui l'exerce: outre les multiples contacts directs ou indirects qu'elle permet avec les personnes, cette fonction maintient un rythme de travail toujours renouvelé et des informations d'actualité particulièrement lors des rencontres internationales.

Pourtant c'est aussi une fonction stressante puisqu'elle comporte des risques: une mauvaise traduction peut occasionner des incidents voire des problèmes diplomatiques.

\section{Les représentations des étudiants}

La majorité des étudiants interrogés répondent: «j'aime ça » ou «j'adore»; on peut voir là un côté «motivation» très prononcé qui semble se limiter à ce simple sentiment «d'aimer la traduction»; ceci pourrait s'expliquer par la satisfaction d'être en mesure de parler plusieurs langues, auto-satisfaction légitime certes.

Mais, en fouillant davantage, on s'aperçoit que le côté lucratif n'est pas négligé, de même que le côté «contact avec les personnalités»; on vous rétorquera: "Dans la conjoncture actuelle, les relations, ça compte».

Enfin, on décèlera également chez les étudiants l'argument «on voyage beaucoup »; une recherche de l'exotisme certes, cette envie de "l'ailleurs» qui sûrement est meilleure que le hic et nunc de l'Algérie actuelle.

\section{Les difficultés}

Les difficultés apparaissent sous des aspects différents selon que la personne est interprète (traduction orale) ou traducteur (traduction par écrit). En effet, «traduire» 
semble plus aisé parce que le traducteur a tout le loisir de consulter documents, ouvrages et dictionnaires spécialisés, tandis que l'interprète doit traduire instantanément à la fin de la lecture d'un discours.

Hormis cet aspect concernant la spécialisation du traducteur, la nature même de la traduction varie en fonction des textes à traduire.

Si le transcodage et la traduction automatique sont possibles dans la traduction des textes scientifiques, techniques et spécialisés (tels que les textes de lois et les textes administratifs), ils deviennent inopérants lorsque l'on aborde les textes littéraires et les textes poétiques même si G. Mounin a pu écrire: "Il est possible de traduire non seulement parce que les hommes par delà l'extrême diversité des langues partagent fondamentalement une même expérience ou connaissance du monde, ce qui rend possible la communication de celle-ci, mais encore même parce qu'il existe des universaux poétiques substantiels.» On remarquera que la traduction véritable devient compliquée au fur et à mesure de la traduction du plus ou moins figé, tels que les proverbes, les maximes ou tout simplement les expressions figées. Ce pas que le traducteur doit franchir en passant d'une culture à une autre est l'écueil le plus redoutable. Pour illustrer notre propos, un exemple est nécessaire; on dira:

- cette nouvelle m'a réchauffé le cœur

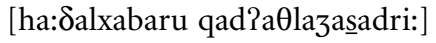

La pertinence réside dans le fait que par delà les structures formelles et culturelles particulières à chaque langue et à chaque civilisation, il serait possible de rendre dans toutes les langues un même «effet», c'est précisément là où la dextérité du traducteur doit prendre toute sa mesure.

\section{Conclusion}

Après cet itinéraire de la traduction en Algérie, il est permis de s'interroger sur l'efficacité de l'École d'Interprétariat et de Traduction. Peut-on se poser la question de sa performance au plan quantitatif? On sait que les ouvrages traduits restent indéniablement en deçà des besoins. La majorité des utilisateurs d'ouvrages, revues, et dictionnaires spécialisés souffrent de ce manque. Les équipes normalement habilitées pour ce type de travaux n'existent pas. Elles relèvent surtout de pratiques ponctuelles. Où réside la solution? Des maisons d'édition commencent à foisonner dans le pays, auront-elles la sagacité de trouver le filon salvateur: un choix difficile s'impose entre la priorité de la recherche de la connaissance et celle du lucre.

\section{RÉFÉRENCES}

El Foul, L. (1996): «Traduction et enseignement des langues», Cahiers de traduction, 2, Université d'Alger, 1996.

Hellat, Y. (1986): La théorie de la traduction, Alger, OPU.

— (1993): "Quelques généralités sur le texte en pédagogie de la traduction», Cahiers de traduction, 1, Université d'Alger.

Institut d'Interprétariat et de traduction: Guide de l'étudiant, mars 1995.

Ladmiral, J. R.: Traduire: Théorèmes pour la traduction, Petite bibliothèque Payot, Paris 1987.

Mounin, G.: Les problèmes théoriques de la traduction, Université Val-de-Marne-Créteil, diffusion honoré Champion, Paris, 1980 ( $2^{\mathrm{e}}$ édition).

Redounne, J.: La traductologie, OPU, Alger 1985. 